\title{
Knockdown of long noncoding RNA Malat1 aggravates hypoxia-induced cardiomyocyte injury by targeting miR-217
}

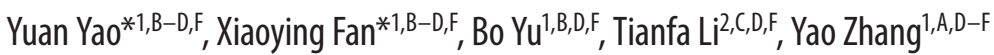 \\ ${ }^{1}$ Department of Cardiovascular Medicine, The $2^{\text {nd }}$ Affiliated Hospital of Harbin Medical University, China \\ ${ }^{2}$ Department of Cardiovascular Medicine, The Affiliated Hospital of Hainan Medical College, Haikou, China \\ A - research concept and design; $B$ - collection and/or assembly of data; $C$ - data analysis and interpretation; \\ $D$ - writing the article; $E$ - critical revision of the article; $F$ - final approval of the article
}

\section{Address for correspondence \\ Yao Zhang \\ E-mail:zhangya00612@126.com}

Funding sources

None declared

\section{Conflict of interest}

None declared

* These authors contributed equally to this work.

Received on May 5, 2017

Reviewed on June 30, 2017

Accepted on August 1, 2018

Published online on March 7, 2019

\begin{abstract}
Background. Expression of long noncoding (IncRNA) Malat1 can be increased by hypoxia in cardiomyocyte. Downregulation of Malat1 contributes to the reduction of cardiomyocyte apoptosis. However, the function of Malat1 in myocardial ischemia is unclear.

Objectives. This study investigated the functional role of IncRNA Malat1 in hypoxia-induced H9c2 cell injury.

Material and methods. H9c2 cells were exposed to hypoxia treatment. Cell proliferation, migration, invasion, and apoptosis were detected using trypan blue exclusion assay, two-chamber migration/invasion assay, annexin V-FITC/PI staining, and western blotting, respectively. Quantitative real-time polymerase chain reaction (GRT-PCR) was performed to analyze the expression levels of Malat1. The effects of Malat1 knockdown on cell proliferation, migration, invasion, and apoptosis were also measured. The interaction between Malat1 and microRNA-217 (miR-217) as well as miR-217 and sirtuin 1 (Sirt1) were analyzed using a dual luciferase reporter assay and qRT-PCR. Effects of miR-217 and Sirt1 on hypoxia-induced H9c2 cell growth were assessed.

Results. Hypoxia induced H9C2 cell injury by inhibiting cell proliferation, migration and invasion, and by promoting apoptosis. Hypoxia significantly enhanced the expression of Malat1. Malat1 bound to miR-217 and Sirt1 was a direct target of miR-217. Knockdown of Malat1 aggravated hypoxia-induced H9C2 cell injury by overexpression of miR-217. Overexpression of Sirt 1 alleviated H9C2 cell injury by activating phosphatidylinositol 3-kinase/protein kinase 3 (PI3K/AKT) and Notch signaling pathways.
\end{abstract}

Conclusions. These findings suggest that Malat1 exerted important roles in hypoxia-induced cardiomyocyte injury by regulating miR-217-mediated Sirt1 and downstream PIBK/AKT and Notch signaling pathways.

Key words: myocardial ischemia, Malat1, hypoxia-induced cell injury, microRNA-217, sirtuin 1

Cite as

Yao Y, Fan X, Yu B, Li T, Zhang Y. Knockdown of long noncoding RNA Malat1 aggravates hypoxia-induced cardiomyocyte injury by targeting miR-217. Adv Clin Exp Med. 2019;28(6):719-728. doi:10.17219/acem/93878

D0I

$10.17219 /$ acem $/ 93878$

Copyright

Copyright by Author(s)

This is an article distributed under the terms of the

Creative Commons Attribution Non-Commercial License

(http://creativecommons.org/licenses/by-nc-nd/4.0/) 


\section{Background}

Myocardial ischemia is a heart condition caused by the reduction of blood flow to the heart, which prevents the heart from receiving enough oxygen. ${ }^{1}$ It has become one of the leading causes of death all over the world and a serious threat to human health. ${ }^{2}$ Myocardial ischemia is the main factor contributing to cardiomyocyte impairment. ${ }^{3}$ The main clinical symptoms of myocardial ischemia are severe persistent chest pain, dyspnea, fever, and syncope. ${ }^{4,5}$ Hypoxia in varying degrees threatens the function and survival of cardiomyocytes, ${ }^{6,7}$ although numerous adaptive countermeasures can be activated in the cardiomyocytes in response to the hypoxic condition. ${ }^{8,9}$

Recently, researchers have demonstrated that numerous noncoding transcripts were involved in the physiological and pathological regulation of many diseases, including myocardial ischemia. ${ }^{10,11}$ Long noncoding RNAs (lncRNAs) are noncoding RNA molecules longer than 200 nucleotides that are not translated into proteins, but regulate the transcription of genes that are involved in different cellular processes, including differentiation, cancer initiation and progression. ${ }^{12}$ Metastasis-associated lung adenocarcinoma transcript 1 (Malat1), an lncRNA, is expressed in the nucleus and participates in many cellular processes. ${ }^{13}$ Several studies have confirmed that Malat1 is responsible for cancer. ${ }^{14-16}$ Zhao et al. proved that Malat1 functions as a mediator in the cardioprotective effects of fentanyl on myocardial ischemiareperfusion injury. ${ }^{17}$ More research is still needed to further explore the effects of Malat1 on myocardial ischemia.

Several lncRNAs or microRNAs (miRNAs) are functionally involved in acute myocardial infarction (e.g., ANRIL, KCNQ1OT1, Malat1, miR-499, and miR-214), mitochondrial function and apoptosis of cardiomyocytes (e.g., CHRF, miR-145 and miR-22). ${ }^{18-24}$ Although lncRNAs have not been extensively researched in myocardial ischemic injury, recent studies are increasingly focusing on the possible contribution of lncRNAs in ischemia. ${ }^{25,26}$ Michalik et al. demonstrated that Malat1 regulates the endothelial cell function and vessel growth. ${ }^{27}$ Interestingly, a negative correlation exists between Malat1 and miR-217. ${ }^{13}$ Moreover, researchers found that $\mathrm{miR}-217$ targets and regulates sirtuin 1 (Sirt1) expression. ${ }^{28}$ However, involvement and the functional mechanism of miR-217 and Malat1 in myocardial ischemic injury remains uncertain. The present study established an in vitro myocardial cell model of hypoxia and investigated the effects of Malat1, miR-217 and Sirt1 on the hypoxia-induced cardiomyocyte injury.

\section{Material and methods}

\section{Cell culture and treatment}

The cardiomyocytes cell line $\mathrm{H} 9 \mathrm{c} 2$ was purchased from Sigma-Aldrich (St. Louis, USA) and cultured in Dulbecco's
Modified Eagle Medium (DMEM; Life Technologies Corp., Carlsbad, USA) supplemented with $10 \%$ fetal bovine serum (FBS), 1\% penicillin/streptomycin (100 U/mL:100 mg/mL) and $1 \%$ GlutaMAX (Life Technologies), at $37^{\circ} \mathrm{C}$ under $5 \%$ $\mathrm{CO}_{2}$. Culture medium was changed every other day. $\mathrm{H} 9 \mathrm{c} 2$ cells were cultured in a hypoxic incubator with $3 \% \mathrm{O}_{2}$ concentration for $24 \mathrm{~h}$ to induce injury.

\section{Quantitative real-time polymerase chain reaction}

After relevant treatment, total RNA was extracted from cells using Trizol reagent (Life Technologies) according to the manufacturer's instructions. Quantitative real-time polymerase chain reaction analysis was performed using One Step SYBR PrimeScript PLUS RT-RNA PCR Kit (TaKaRa Biotechnology, Dalian, China) to assess the expression levels of Malat1 according to the protocol instructions. Taqman MicroRNA Reverse Transcription Kit and Taqman Universal Master Mix II with the TaqMan MicroRNA Assay of miR-217 and U6 (Applied Biosystems, Foster City, USA) were used to test the expression levels of miR-217 in cells. RNA PCR Kit (AMV) v. 3.0 (TaKaRa Biotechnology) was used to assess the expression of Sirt1. GAPDH or U6 was used as an internal control. The relative expressions were calculated using $2^{-\Delta \Delta \mathrm{Ct}}$ method. Sequences of primers were as follows: Malat1 forward primer: 5-AGCGGAAGAACGAATGTAAC-'3, reverse primer: 5'-GAACAGAAGGAAGAGCCAAG-'3; miR-217 forward primer: 5'TACTGCATCAGGAACTGACTGGA-'3, reverse primer: 5'-GTGCAGGGTC CGAGGT-'3; Sirt1 forward primer: 5'-AGGAGACTTGCCTGGTGAAA-'3, reverse primer: 5'-CAGGGGTGGTTATTGCATCT-'3; GAPDH forward primer: 5'-GCACCGTCAAGGCTGAGAAC-'3, reverse primer: 5'-TGGTGAAGACGC CAGTGGA-'3; U6 forward primer: 5'CTCGCTTCGGCAGCACATATACT-'3, reverse primer: 5'-ACGCTTCACGAATTTGCGTGTC-'3.

\section{Transfection and generation of stably transfected cell lines}

Short-hairpin RNA directed against lncRNA Malat1 was ligated into the U6/GFP/Neo plasmid (GenePharma, Shanghai, China) and this was referred to as sh-Malat1. The following target regions were chosen: Malat1\#1, 5'-GGGAGTTACTTGCCAACTTG-'3; Malat1\#2, 5'-CCAGGCTGGTTATG ACTCAG-'3. For the analysis of Sirt1 functions, full-length Sirt1 sequences and short-hairpin RNA directed against Sirt1 were constructed in pEX-2 and U6/GFP/Neo plasmids (GenePharma), respectively. These were referred to as $\mathrm{pEX}$-Sirt1 and sh-Sirt1. The sequence of pEX-Sirt1 was 5'-ACUUUGCUGUAACCCUGUA-'3. The forward sequence of sh-Sirt1 was 5'-CACCACACCAGATTCTTCAGTGAT TGTCATCTCTGACAATCACTGAAGAATCTGGTGG-'3 and the reverse sequence of sh-Sirt1 was 5'-AAAACCAGATTCTTCAGTG 
ATTGTCAGAGATGA CAATCACTGAACCTGGTGG-'3. The plasmid carrying a non-targeting sequence was used as a negative control (NC) that was referred to as sh-NC. miR-217 mimics, inhibitors and their respective NCs were synthesized by Life Technologies and were transfected into the cells in line with the manufacturer's instruction. The sequences used were: pEX-miR-217, 5'-UACUGCAUCAGGAACUGAUUGGA-'3; si-miR-217, 5'-UACUGCAUCAGG AACUGAUUGGA-'3. Cell transfection was performed using lipofectamine 3000 reagent (Life Technologies) according to the manufacturer's instructions. The stably transfected cells were selected by the culture medium containing $0.5 \mathrm{mg} / \mathrm{mL}$ of G418 (Sigma-Aldrich). After approx. 4 weeks, G418-resistant cell clones were established. The highest transfection efficiency occurred at $48 \mathrm{~h}$, and thus $72 \mathrm{~h}$ post-transfection was considered as the harvest time in the subsequent experiments.

\section{Cell proliferation assays}

For cell proliferation assay, $1 \times 10^{5}$ cells were seeded in duplicate in 60-millimeter dishes. After normoxia or hypoxia treatment for $24 \mathrm{~h}$, cells were washed and the live cell numbers were determined using trypan blue exclusion assay.

\section{Apoptosis assay}

Cell apoptosis assay was performed using propidium iodide (PI) and fluorescein isothiocynate (FITC)-conjugated annexin $\mathrm{V}$ staining. Briefly, $1 \times 10^{5}$ cells were seeded in duplicate in 60-millimeter dishes. After normoxia or hypoxia treatment for $24 \mathrm{~h}$, the cells were washed in phosphate buffered saline (PBS) and fixed in 70\% ethanol. Fixed cells were then washed twice in PBS and stained with PI/FITC-annexin $\mathrm{V}$ in the presence of $50 \mu \mathrm{g} / \mathrm{mL}$ RNase A (Sigma-Aldrich), and then were incubated for $1 \mathrm{~h}$ at room temperature in the dark. The rate of apoptotic cells was recorded using Flow cytometer (Beckman Coulter, Fullerton, USA). The data was analyzed using Flowjo software (www.flowjo.com).

\section{Migration and invasion assay}

Cell migration was determined using a modified twochamber migration assay with a pore size of $8 \mathrm{~mm}$. After normoxia or hypoxia treatment for $24 \mathrm{~h}, 5.0 \times 10^{4} \mathrm{H} 9 \mathrm{c} 2$ cells were seeded into the upper compartment of 24-well transwell culture chamber supplemented with $200 \mathrm{~mL}$ of serum-free medium, while $600 \mathrm{~mL}$ of complete medium was added into the lower compartment. After incubation at $37^{\circ} \mathrm{C}$ for $48 \mathrm{~h}$, the cells were fixed with methanol. After that, non-traversed cells were carefully removed from the upper surface of the filter with a cotton swab, while traversed cells on the lower side of the filter were stained with crystal violet and then counted.

The invasive behavior of the cells was determined using 24-well Millicell Hanging Cell Culture inserts with $8 \mathrm{~mm}$
PET membranes (Merck Millipore, Bedford, USA). In brief, after normoxia or hypoxia treatment for $24 \mathrm{~h}, 5.0 \times 10^{4}$ H9c2 cells in $200 \mu \mathrm{L}$ serum-free DMEM medium were plated onto BD BioCoat Matrigel Invasion Chambers $(8 \mu \mathrm{M}$ pore size polycarbonate filters; BD Biosciences, Franklin Lakes, USA), while a complete medium containing 10\% FBS was added to the lower chamber. After processing the invasion chambers for $48 \mathrm{~h}\left(37^{\circ} \mathrm{C}, 5 \% \mathrm{CO}_{2}\right.$ or $37^{\circ} \mathrm{C}$, $3 \% \mathrm{CO}_{2}$ ) in accordance with the manufacturer's protocol, the non-invading cells were removed with a cotton swab. The invading cells were fixed in $100 \%$ methanol and then stained with crystal violet solution and counted microscopically. The data is presented as the average number of cells attached to the bottom surface from 5 randomly chosen fields.

\section{Reporter vector constructs and luciferase reporter assay}

The Malat1 fragment containing the predicted miR-217 binding site was amplified using PCR and then cloned into pmirGlO Dual-Luciferase miRNA Target Expression Vector (Promega, Madison, USA) to form the reporter vector Malat1-wild-type (Malat1-Wt). To mutate the putative binding site of miR-217 in the Malat1, the sequence of putative binding site was replaced and named as Malat1mutated-type (Malat1-Mt). Then the vectors and miR217 mimics were co-transfected into HEK293 cells and the Dual-Luciferase Reporter Assay System (Promega) was performed to test the luciferase activity.

The construction process of Sirt1-wt and Sirt1-mt reporter vectors was similar to the Malat1-wt and Malat1mt. After that, Sirt1-wt and Sirt1-mt reporter vectors and miR-217 mimic were co-transfected into HEK293 cells, and the relative luciferase activities were measured with Dual-Luciferase Reporter Assay System.

\section{Western blotting}

The protein used for western blotting was extracted using the RIPA lysis buffer (Beyotime Biotechnology, Shanghai, China) that was supplemented with protease inhibitors (Roche Diagnostics, Basel, Switzerland). The proteins were then quantified using the BCA Protein Assay Kit (Pierce, Appleton, USA) accordingly. The western blotting system was established using a Bio-Rad Bis-Tris Gel system (Bio-Rad Laboratories, Hercules, USA), according to the manufacturer's instructions. Protein samples were electrophoresed using western blot system and transferred into polyvinylidene difluoride (PVDF) membranes (Merck Millipore). After being blocked with 5\% bovine serum albu$\min$ (BSA; Sigma-Aldrich) for $1 \mathrm{~h}$, the membrane was incubated with primary antibodies at $4^{\circ} \mathrm{C}$ overnight. The following primary antibodies were used in this study: anti-Bcl-2 antibody (ab59348), anti-Bax antibody (ab182733), anti-pPI3K antibody (ab191606), anti-PI3K antibody (ab109006), 
anti-p-AKT antibody (ab81283), anti-AKT antibody (ab8805), and anti-Notch 3 (ab23426); all of the above were obtained from Abcam Biotechnology (Cambridge, UK). Anti-caspase 3 antibody (\#9662), anti-caspase 9 antibody (\#9508), anti-Notch 1 antibody (\#3608), anti-Notch 2 antibody (\#5732), and anti-GAPDH antibody (\#5174) were purchased from Cell Signaling Technology (Danvers, USA). After that, membranes were washed and incubated with secondary antibody (ab6788, ab6721; Abcam Biotechnology) for $1 \mathrm{~h}$ at room temperature. The protein signals were measured using Bio-Rad ChemiDoc XRS system (Bio-Rad), which was supplemented with $200 \mu \mathrm{L}$ of Immobilon Western Chemiluminescent HRP Substrate (Merck Millipore).

\section{Statistical analysis}

All experiments were repeated 3 times. The results of multiple experiments are presented as mean \pm standard deviation (SD). Statistical analyses were performed using Graphpad v. 6.0 statistical software (Graphpad Software, San Diego, USA). The p-values were calculated using oneway analysis of variance (ANOVA) with Sidak post-hoc test. A p-value $<0.05$ was considered to indicate a statistically significant result.

\section{Results}

\section{Hypoxia induces H9c2 cell injury}

Hypoxic treatment significantly downregulated the $\mathrm{H} 9 \mathrm{c} 2$ cell viability (Fig. 1A, p < 0.05), migration (Fig. 1B, p < 0.05) and invasion (Fig. $1 C, \mathrm{p}<0.05$ ), while increasing cell apoptosis (Fig. 1D, p < 0.005) as compared to the normoxia treatment. Similar results were obtained from western blotting analysis (Fig. 1E), which presented that the expression levels of Bax, cleaved-caspase 3 and cleaved-caspase 9 were all enhanced after hypoxia treatment. These results suggest that hypoxia induces $\mathrm{H} 9 \mathrm{c} 2$ cell injury by inhibiting cell viability, migration and invasion, and promoting cell apoptosis.

\section{Hypoxia promotes the expression of Malat1}

The qRT-PCR was performed to analyze the expression level of Malat1 after hypoxia treatment. Results showed that the relative expression of Malat1 was significantly increased in the hypoxic condition compared to the control (Fig. 2, p < 0.01).

\section{Suppression of Malat1 aggravates hypoxia-induced H9c2 cell injury}

Either sh-Malat1\#1 or sh-Malat1\#2 were transfected into H9c2 cells to decrease the expression level of Malat1. As shown in Fig. 3A, the relative expression of Malat1 was significantly decreased in H9c2 cells after transfection with sh-Malat1\#1 ( $<$ < 0.005) and sh-Malat1\#2 ( $<0.01)$. Considering that sh-Malat1\#1 had more significant inhibition, sh-Malat\#1 was used in further experiments. Hypoxia-induced cell injury was significantly exacerbated by Malat 1 suppression, as evidenced by cell viability (Fig. 3B, p < 0.05), migration (Fig. 3C, p < 0.05) and invasion (Fig. 3D, p < 0.05) decreases, and by cell apoptosis increase (Fig. 3E, p < 0.01) after sh-Malat1\#1 transfection. The apoptosis results were further confirmed with western blotting (Fig. 3F), which indicted that the expressions of Bax, cleaved-caspase 3 and cleaved-caspase 9 were further upregulated after Hypoxia+sh-Malat1\#1 treatment. The expression of Bcl-2 was downregulated after Hypoxia+sh-Malat1\#1 treatment. These results suggest that knockdown of Malat1 expression aggravates hypoxiainduced $\mathrm{H} 9 \mathrm{c} 2$ cell injury.

\section{Malat1 negatively regulates the level of miR-217 in H9c2 cells}

The qRT-PCR was performed to detect the expression of miR-217 in H9c2 cells after hypoxia treatment and shMalat1\#1 transfection. As shown in Fig. 4A, the expression of miR-217 was significantly decreased after hypoxia treatment ( $\mathrm{p}<0.01)$. Knockdown of Malat1 markedly increased the miR-217 expression ( $\mathrm{p}<0.005)$, which suggests that Malat1 bind to miR-217 in $\mathrm{H} 9 \mathrm{c} 2$ cells. This hypothesis was further confirmed by dual luciferase activity assay, which pointed out co-transfection with miR-217 and Malat1-wt notably reduced the relative luciferase activity (Fig. 4B, p < 0.05). This data indicates that Malat1 negatively regulates the expression level of miR217 in H9c2 cells.

\section{Knockdown of Malat1 aggravates hypoxia-induced $\mathrm{H} 9 \mathrm{c} 2$ cell injury by upregulating of miR-217}

The effects of Malat1 and miR-217 on viabilities, migration, invasion, and apoptosis of $\mathrm{H} 9 \mathrm{c} 2$ cells were then investigated. The expression level of miR-217 was significantly increased after miR-217 mimic transfection (Fig. 5A, $\mathrm{p}<0.005)$ and remarkably decreased after si-miR-217 transfection $(\mathrm{p}<0.01)$. The si-miR-217 single transfection obviously reversed the hypoxia-induced cell viability, migration and invasion inhibition (Fig. 5B-D, p < 0.05) as well as the cell apoptosis enhancement (Fig. 5E, $\mathrm{p}<0.01$ ). Moreover, compared to hypoxia+sh-Malat $\# 1+$ siNC treatment group, the cell viability, migration and invasion were all increased in hypoxia+sh-Malat1\#1+si-miR-217 treatment group (Fig. 5B-D, p < 0.01). The cell apoptosis was dramatically decreased after hypoxia+sh-Malat $1 \# 1+$ simiR-217 treatment (Fig. 5E, p < 0.005). This data suggests that knockdown of Malat1 aggravates hypoxia-induced H9c2 cell injury by overexpression of miR-217. 
A

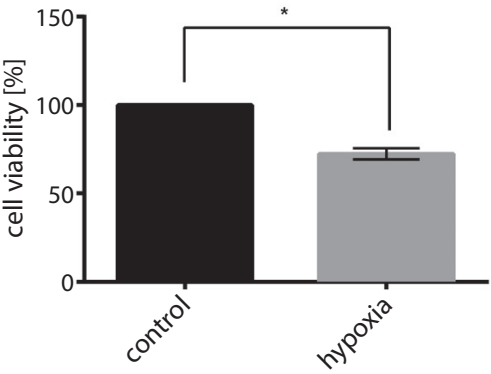

C

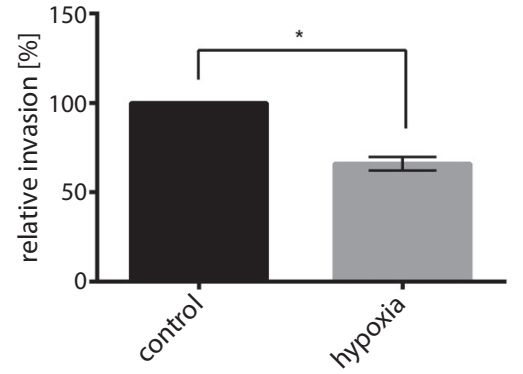

E

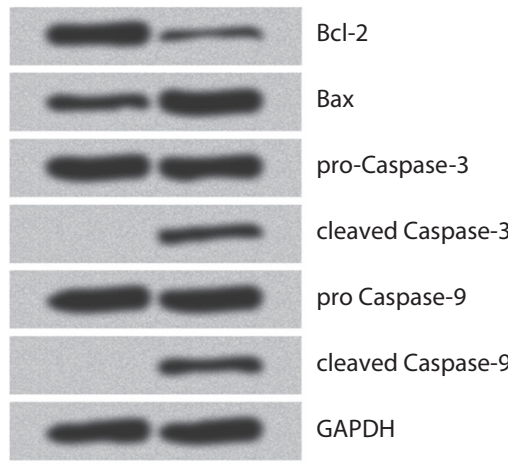

control hypoxia
B

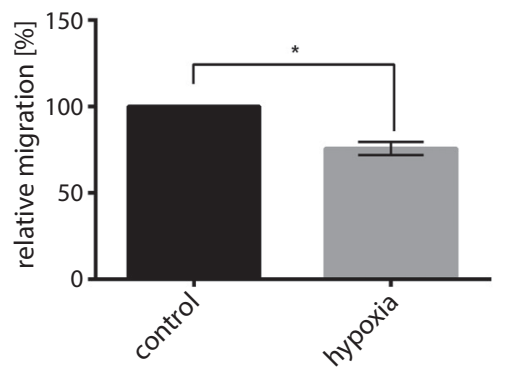

D

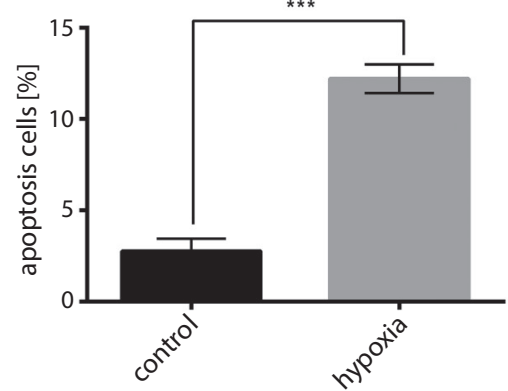

Fig. 1. Effects of hypoxia on H9c2 cell viability, migration, invasion, and apoptosis. Cell viability (A), migration (B), invasion (C), and apoptosis (D) after normoxia or hypoxia treatment was detected using trypan blue exclusion, migration and invasion, and annexin V-FITC/PI staining, respectively. (E) Western blotting was used to analyze the expression levels of $\mathrm{Bcl}-2$, Bax, caspase 3 , and caspase 9 in $\mathrm{H} 9 \mathrm{c} 2$ cells after normoxia or hypoxia treatment. ${ }^{*} p<0.05$; ${ }^{* * *} p<0.005$

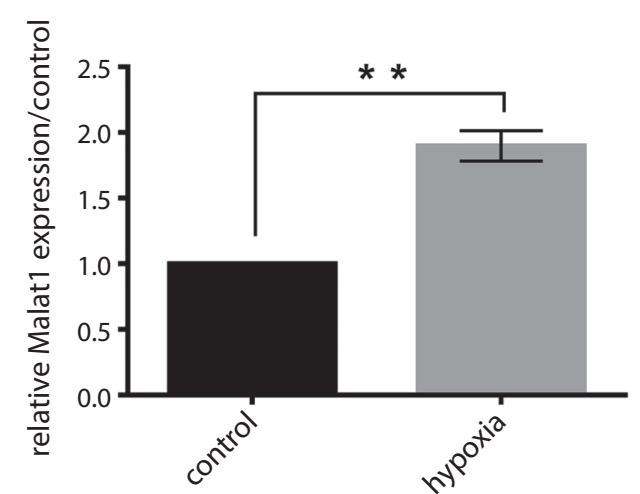

Fig. 2. Effect of hypoxia on expression of Malat1 in $\mathrm{H} 9 \mathrm{c} 2$ cells. Quantitative real-time polymerase chain reaction (qRT-PCR) was performed to measure the expression level of Malat1 in H9c2 cells after hypoxia treatment. ${ }^{* *} p<0.01$

\section{miR-217 negatively regulates the expression of Sirt1 in H9c2 cells}

Sirt1 was hypothesized to be a potential target of miR217. As shown in Fig. 6A, the expression level of Sirt1 was remarkably upregulated after hypoxia treatment $(\mathrm{p}<0.01)$.
Moreover, the expression of Sirt1 was obviously downregulated after miR-217 mimic transfection $(\mathrm{p}<0.005)$ and noticeably upregulated after si-miR-217 transfection $(\mathrm{p}<0.01)$, which suggests that miR-217 negatively regulated the expression of Sirt1 in H9c2 cells. To verify whether miR-217 was able to directly bind to the 3' untranslated region (3'UTR) of Sirt1, Sirt1-wt and Sirt1-mt containing the wild-type and mutant binding sequences of miR-217 were generated, respectively (Fig. 6B). A luciferase reporter assay revealed that the relative luciferase activity was significantly reduced when co-transfected with Sirt1-wt and miR-217 mimics $(\mathrm{p}<0.05)$. However, the luciferase activity revealed no significant difference when co-transfected with Sirt1-mt and miR-217 mimics. These results indicate that Sirt1 was a direct target gene of miR-217 in H9c2 cells.

\section{Overexpression of Sirt1 alleviates the hypoxia-induced $\mathrm{H} 9 \mathrm{c} 2$ cell injury}

To analyze the functions of Sirt1, the full-length Sirt1 sequences and short-hairpin RNA directed against Sirt1 were constructed in pEX-2 and U6/GFP/Neo plasmids, respectively. They were referred to as pEX-Sirt1 and sh-Sirt1. 
A

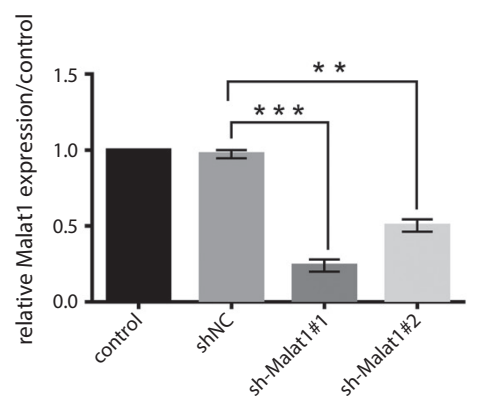

C

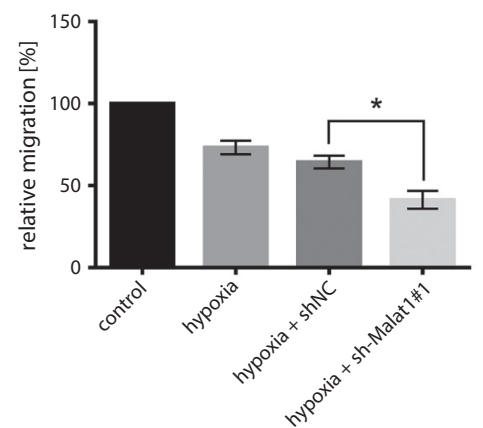

E

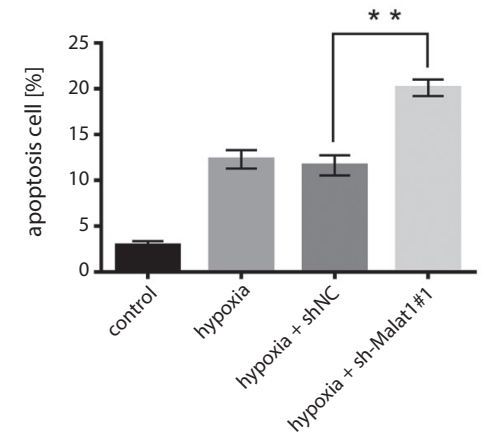

A

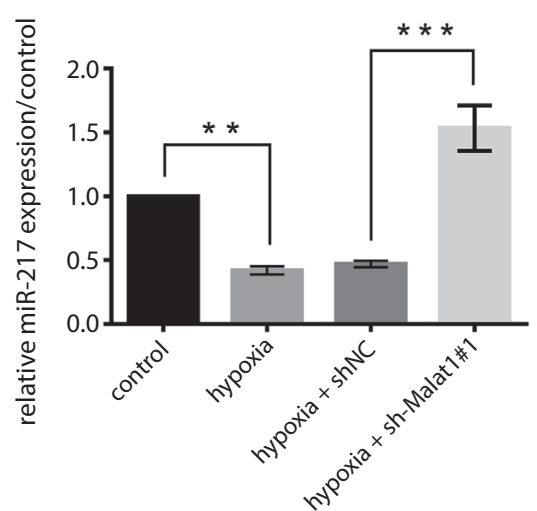

B

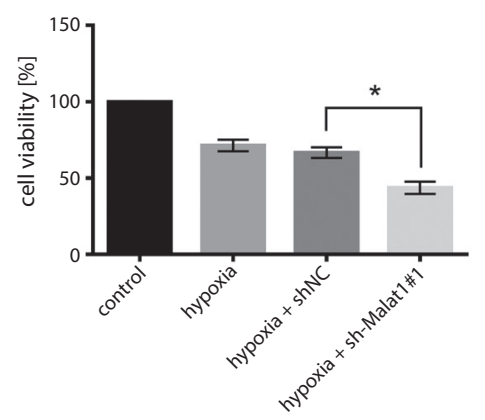

D

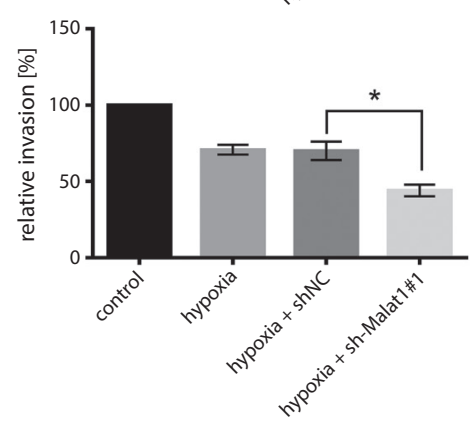

$\mathbf{F}$

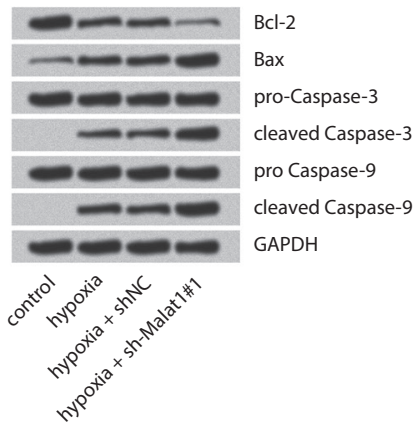

Fig. 3. Effects of suppression of Malat1 on hypoxiainduced $\mathrm{H} 9 \mathrm{c} 2$ cell injury. (A) quantitative real-time polymerase chain reaction (qRT-PCR) was used to detect the expression level of Malat1 after shMalat1\#1 or sh-Malat1\#2 transfection. Cell viability (B), migration (C), invasion (D), and apoptosis (E) after hypoxia treatment and/or sh-Malat1 transfection were measured using trypan blue exclusion, migration and invasion, and annexin V-FITC/PI staining, respectively. (F) Western blotting was performed to analyze the expression levels of $\mathrm{BCl}-2$, Bax, caspase 3, and caspase 9 in $\mathrm{H} 9 \mathrm{c} 2$ cells after hypoxia treatment and/or sh-Malat1 transfection. ${ }^{*} p<0.05 ;{ }^{* *} p<0.01 ;{ }^{* *} p<0.005$

B

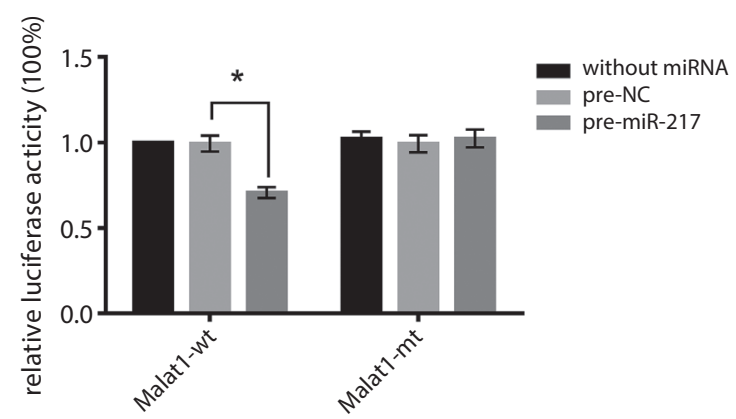

Fig. 4. The relationship between Malat1 and miR-217. (A) The expressions of miR-217 in H9c2 cells after hypoxia treatment and/or sh-Malat1 transfection were determined using quantitative real-time polymerase chain reaction (qRT-PCR). (B) The relative luciferase activity was detected after co-transfected miR-217 with Malat1-wt or Malati-mt. ${ }^{* *} p<0.01 ; * * p<0.005$

As presented in Fig. 7A, the relative Sirt1 expression was obviously increased after pEX-Sirt1 transfection $(\mathrm{p}<0.05)$ and significantly decreased after sh-Sirt1 transfection $(\mathrm{p}<0.01)$. Overexpression of Sirt1 significantly reversed the hypoxia-induced cell viability, migration and invasion inhibition (Fig. 7B-D, p < 0.05) as well as cell apoptosis enhancement (Fig. 7E, $\mathrm{p}<0.05$ ). As expected, suppression of Sirt1 showed opposite results (Fig. 7B-E). These results suggest that overexpression of Sirt1 protects H9c2 cells from hypoxia-induced injury. 
A

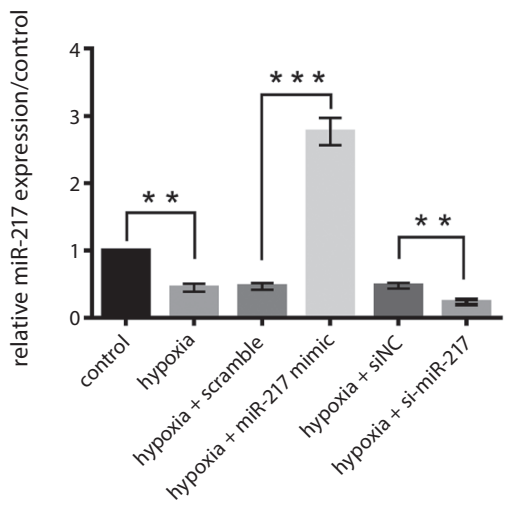

C

$\mathbf{E}$
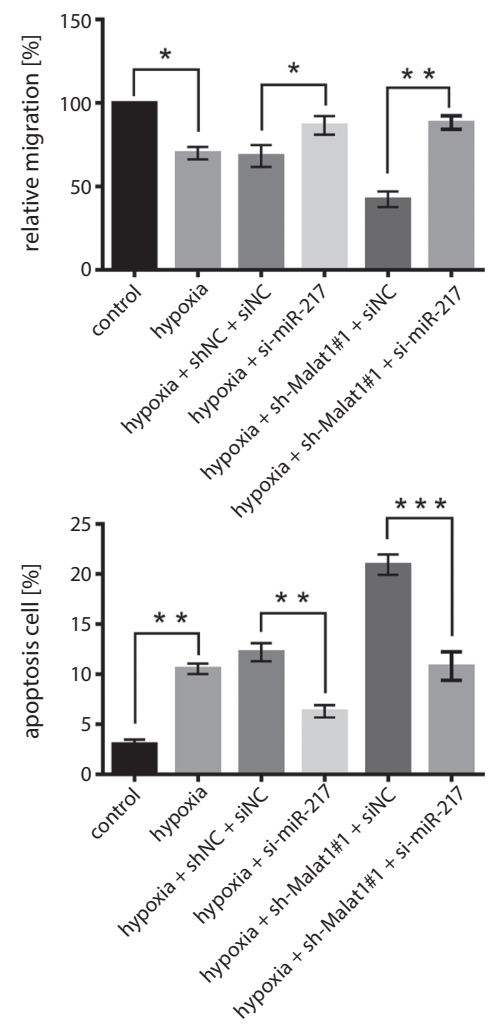

A

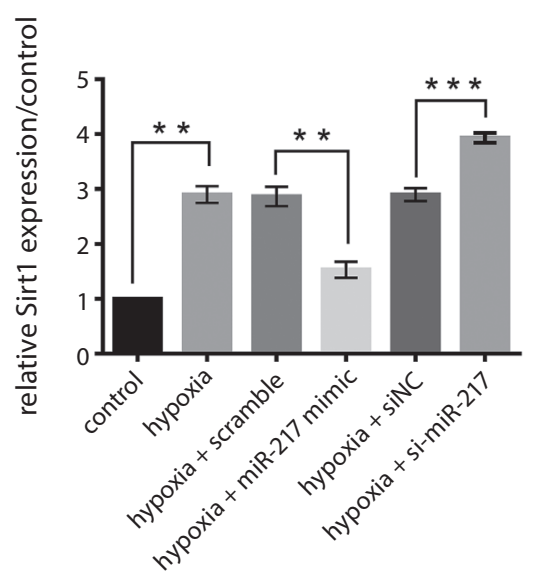

B

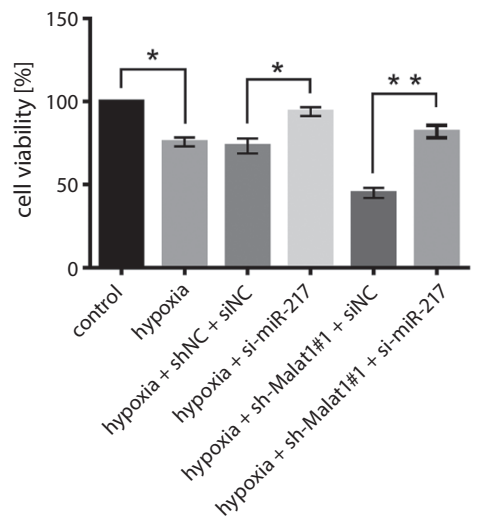

Fig. 5. Roles of miR-217 in hypoxia and sh-Malat1induced $\mathrm{H} 9 \mathrm{c} 2$ cell injury. (A) The expressions of miR-217 were measured after hypoxia treatment and miR-217 mimic or si-miR-217 transfection. Cell viability (A), migration (B), invasion (C), and apoptosis (E) after hypoxia treatment and/or Malat1\#1 as well as si-miR-217 transfection were detected using trypan blue exclusion, migration and invasion, and annexin V-FITC/PI staining, respectively. ${ }^{*} p<0.05$;

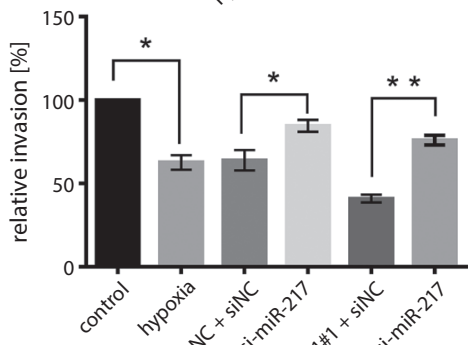

$p<0.01 ;{ }^{* * *} p<0.005$
B

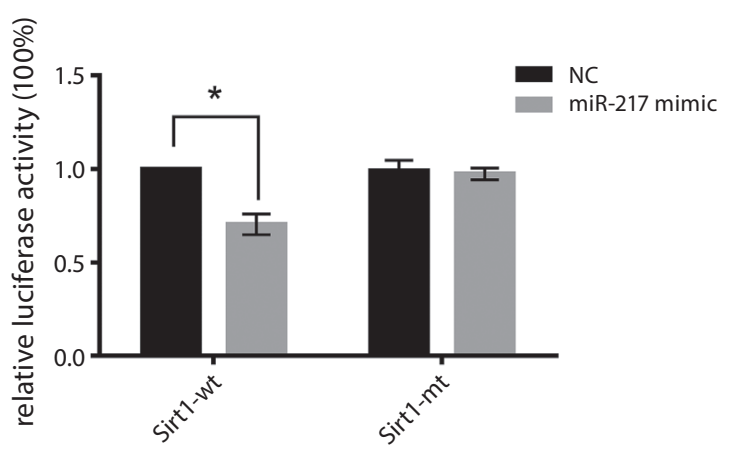

Fig. 6. The relationship between miR-217 and Sirt1. (A) Quantitative real-time polymerase chain reaction (qRT-PCR) was performed to detect the expressions of Sirt1 in H9c2 cells after hypoxia treatment and/or miR-217 mimic/si-miR-217 transfection. (B) The relative luciferase activity was measured after cotransfection with miR-217 mimic and Sirt1-wt or Sirt1-mt. ${ }^{* *} p<0.01 ;{ }^{* * *} p<0.005$ 


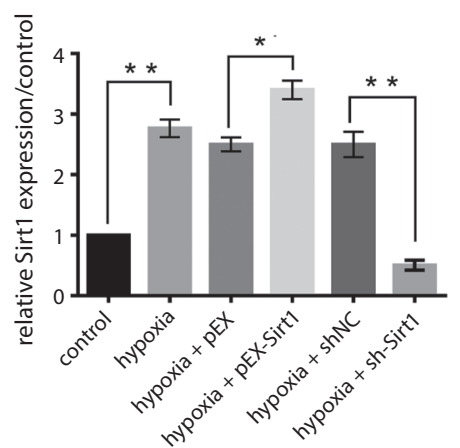

C
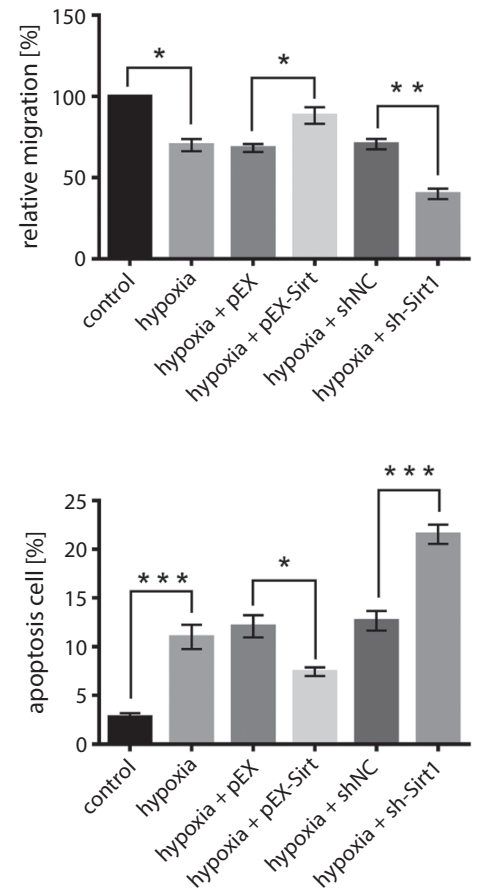

A

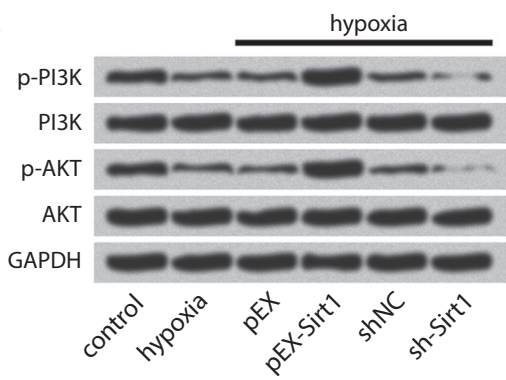

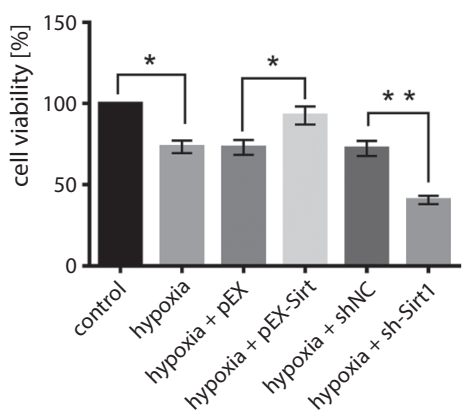

D

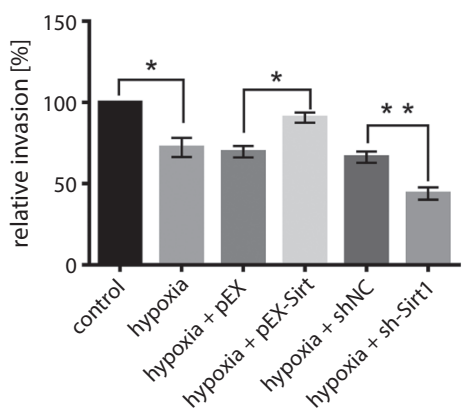

B

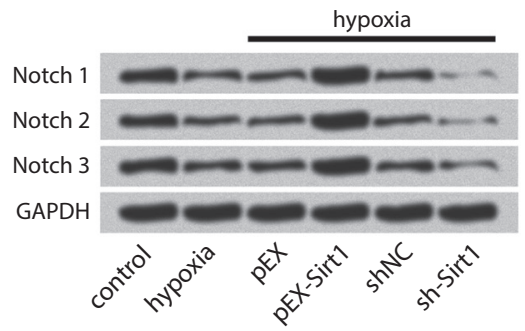

Fig. 7. Effects of Sirt1 on hypoxia-induced H9c2 cell injury. (A) The expressions of Sirt1 in H9c2 cells were detected after hypoxia treatment and/or pEX-Sirt1/ sh-Sirt1 transfection. Cell viability (B), migration (C), invasion (D), and apoptosis (E) after hypoxia treatment and/or pEX-Sirt1/sh-Sirt1 transfection were measured using trypan blue exclusion, migration and invasion, and annexin V-FITC/PI staining, respectively. ${ }^{*} \mathrm{p}<0.05$ ${ }^{* *} \mathrm{p}<0.01 ;{ }^{* *} \mathrm{p}<0.005$

\section{PI3K/AKT and Notch signaling pathways}

Western blot analysis was performed to analyze the roles of PI3K/AKT and Notch signaling pathways in hypoxiainduced $\mathrm{H} 9 \mathrm{c} 2$ cell injury. The results displayed that the expression levels of $\mathrm{p}-\mathrm{PI} 3 \mathrm{~K}$ and $\mathrm{p}-\mathrm{AKT}$ were decreased after hypoxia treatment (Fig. 8A). Overexpression of Sirt1 reversed the hypoxia-induced decreases of $\mathrm{p}-\mathrm{PI} 3 \mathrm{~K}$ and p-AKT but suppression of Sirt1 further aggravated the hypoxia-induced decreases of p-PI3K and p-AKT. Similar results were found in Notch signaling pathway, which showed that the expressions of Notch 1, Notch 2 and Notch 3 were decreased after hypoxia treatment and further downregulated after sh-Sirt1 transfection (Fig. 8B). These results indicate that overexpression of Sirt1 alleviates hypoxia-induced $\mathrm{H} 9 \mathrm{c} 2$ cell injury by activating PI3K/ AKT and Notch pathways.

\section{Discussion}

We studied the effects and mechanisms of Malat1 on the hypoxia-induced injury in $\mathrm{H} 9 \mathrm{C} 2$ cells. We showed that hypoxia induced $\mathrm{H} 9 \mathrm{c} 2$ cell injury by inhibiting cell 
viability, migration and invasion, and promoting cell apoptosis. Suppression of Malat1 aggravates the hypoxia-induced H9c2 cell injury (Fig. 3). Malat1 negatively regulates the expression of miR-217 in H9c2 cells (Fig. 4). Knockdown of Malat1 aggravates hypoxia-induced H9c2 cell injury by overexpression of miR-217 (Fig. 5). Moreover, miR-217 negatively regulates Sirt1 expression and Sirt1 was a target of miR-217 (Fig. 6 and 7). Overexpression of Sirt1 activates PI3K/AKT and Notch signaling pathways, which might be involved in $\mathrm{H} 9 \mathrm{c} 2$ cell survival (Fig. 8).

Hypoxia-induced cell death is a major concern and plays a critical role in various pathophysiological processes, such as hypoxic/ischemic disease, organ transplantation, angiogenesis, or tumor invasion. ${ }^{29-31}$ Cardiomyocyte injury comprises a series of events that may occur together or separately, such as: reperfusion, arrhythmias, myocardial stunning in "reversible mechanical dysfunction", microvascular damage, and cell death. ${ }^{32,33}$

Malat1 was initially discovered as a tumor-associated IncRNA, which is mainly involved in the splicing and epigenetic regulation of gene expression. ${ }^{34}$ A recent study reports that Malat1 also acts as a regulator of cardiovascular disease. ${ }^{35}$ Zhao et al. reported that Malat1 plays a key regulatory role in mediating the cardioprotective effects of fentanyl against ischemic/reperfusion injury. ${ }^{17}$ In our research, we found that the expression of Malat1 in H9c2 cells was significantly increased after hypoxia treatment. Knockdown of Malat1 aggravates hypoxia-induced H9c2 cell injury by enhancing cell viability, migration and invasion inhibition, and promoting cell apoptosis. However, Zhang et al. indicated that downregulation of Malat1 reduced cardiomyocyte apoptosis and improved left ventricular function in diabetic rats. ${ }^{36}$ The regulation of intracellular signaling pathways is very complex. The same molecules may have different regulatory effects in different cell types and in different conditions. The disparity effect of Malat1 on cardiomyocyte cell proliferation and apoptosis might be associated with different cell types and different treatment processes.

Our study indicates that Malat1 negatively regulates the expression of miR-217, which is consistent with another study on human cancers. ${ }^{37}$ Our study is the first to demonstrate that miR-217 binds to Malat1 in cardiomyocytes and the role of miR-217 in cardiomyocyte injury induced by hypoxia. Luciferase activity reveals that miR-217 was able to directly bind to the 3'UTR of Sirt1 and, therefore, Sirt1 was identified as a direct target of miR-217 in H9c2 cells. Several other studies have also confirmed that Sirt1 was a target of miR-217, ${ }^{38}$ which is consistent with our findings.

Sirt1 is a member of a protein family known as sirtuins, which belongs to the Sirt2 family and has been identified as nicotinamide-adenine dinucleotide (NAD) ${ }^{+}$dependent deacetylases. ${ }^{39,40}$ Sirt1 has been demonstrated to participate in cancer, aging, metabolic diseases, and cardiovascular dysfunctions. ${ }^{41,42}$ Sirt1 can protect endothelial cells from oxidative stress and oxidative low-density lipoprotein-induced apoptosis. ${ }^{43-45}$ In our study, we found that overexpression of Sirt1 alleviates the hypoxia-induced $\mathrm{H} 9 \mathrm{c} 2$ cell injury by promoting cell viability, migration and invasion but inhibiting cell apoptosis.

The PI3K/Akt and Notch signaling pathways are essential for cell survival and proliferation. ${ }^{39,46}$ It has been reported that the activation of PI3K/Akt pathway is closely associated with vascular remodeling and angiogenesis. Li et al. demonstrated that Sirt1 promoted the migration and proliferation of endothelial progenitor cells through PI3K/AKT pathway. ${ }^{46}$ Our study results similarly proved that Sirt1 activates PI3K/AKT and Notch signaling pathways, which were involved in the promotion of cell survival in $\mathrm{H} 9 \mathrm{c} 2$ cells.

In conclusion, these findings suggest that knockdown of Malat1 aggravates hypoxia-induced cardiomyocyte injury by upregulating miR-217 expression. The aforementioned results demonstrated negative regulation of Sirt1 expression by miR-217 and Sirt1 as a target of miR-217. Also, Sirt1 activated PI3K/AKT and Notch signaling pathways, which in turn promoted $\mathrm{H} 9 \mathrm{c} 2$ cell survival.

\section{References}

1. Thygesen K, Alpert JS, White HD. Joint ESC/ACCF/AHA/WHF Task Force for the Redefinition of Myocardial Infarction. Universal definition of myocardial infarction. Eur Heart J. 2007;28(20):2525-2538.

2. Zhu HM, Deng L. Evaluation of cardiomyocyte hypoxia injury models for the pharmacological study in vitro. Pharm Biol. 2012;50(2):167-174.

3. Gui L, Liu B, Lv G. Hypoxia induces autophagy in cardiomyocytes via a hypoxia-inducible factor 1-dependent mechanism. Exp Ther Med. 2016;11(6):2233-2239.

4. Coventry LL, Bremner AP, Williams TA, Jacobs IG, Finn J. Symptoms of myocardial infarction: Concordance between paramedic and hospital records. Prehosp Emerg Care. 2014;18(3):393-401.

5. Lippi G, Sanchis-Gomar F, Cervellin G. Chest pain, dyspnea and other symptoms in patients with type 1 and 2 myocardial infarction: A literature review. Int J Cardiol. 2016;215:20-22.

6. Tong W, Xiong F, Li Y, Zhang L. Hypoxia inhibits cardiomyocyte proliferation in fetal rat hearts via upregulating TIMP-4. Am J Physiol Regul Integr Comp Physiol. 2013;304(8):R613-R620.

7. Botting KJ, McMillen IC, Forbes H, Nyengaard JR, Morrison JL. Chronic hypoxemia in late gestation decreases cardiomyocyte number but does not change expression of hypoxia-responsive genes. J Am Heart Assoc. 2014;3(4). doi:10.1161/JAHA.113.000531

8. Ramjiawan A, Bagchi RA, Blant A, et al. Roles of histone deacetylation and AMP kinase in regulation of cardiomyocyte PGC-1a gene expression in hypoxia. Am J Physiol Cell Physiol. 2013;304(11):C1064-1072.

9. Wang W, Peng Y, Wang Y, Zhao X, Yuan Z. The anti-apoptotic effect of heat shock protein 90 on hypoxia-mediated cardiomyocyte damage through the PI3K/Akt pathway. Clin Exp Pharmacol Physiol. 2009;36(9):899-903.

10. Wang K, Liu CY, Zhou LY, et al. APF IncRNA regulates autophagy and myocardial infarction by targeting miR-188-3p. Nat Commun. 2015; 6:6779.

11. Wang JX, Zhang XJ, Li Q, et al. MicroRNA-103/107 regulate programmed necrosis and myocardial ischemia/reperfusion injury through targeting FADD. Circ Res. 2015;117(4):352-363.

12. Reik W. Evolution and functions of long noncoding RNAs. Cell. 2009; 136(4):629-641.

13. Zhang X, Tang X, Liu K, Hamblin MH, Yin KJ. Long non-coding RNA Malat1 regulates cerebrovascular pathologies in ischemic stroke. J Neurosci. 2017;37(7):1797-1806.

14. Lin R, Maeda S, Liu C, Karin M, Edgington TS. A large noncoding RNA is a marker for murine hepatocellular carcinomas and a spectrum of human carcinomas. Oncogene. 2007;26(6):851-858. 
15. Wei Y, Niu B. Role of MALAT1 as a Prognostic factor for survival in various cancers: A systematic review of the literature with meta-analysis. Dis Markers. 2015;2015:164635.

16. Gutschner T, Hammerle M, Eissmann M, et al. The noncoding RNA MALAT1 is a critical regulator of the metastasis phenotype of lung cancer cells. Cancer Res. 2013;73(3):1180-1189.

17. Zhao ZH, Hao W, Meng QT, Du XB, Lei SQ, Xia ZY. Long non-coding RNA MALAT1 functions as a mediator in cardioprotective effects of fentanyl in myocardial ischemia-reperfusion injury. Cell Biol Int. 2016;41(1):62-70.

18. Vausort M, Wagner DR, Devaux Y. Long noncoding RNAs in patients with acute myocardial infarction. Circ Res. 2014;115(7):668-677.

19. Janssen R, Zuidwijk MJ, Muller A, et al. MicroRNA 214 is a potential regulator of thyroid hormone levels in the mouse heart following myocardial infarction, by targeting the thyroid-hormone-inactivating enzyme deiodinase type III. Front Endocrinol (Lausanne). 2016;7:22.

20. Xin Y, Yang C, Han Z. Circulating miR-499 as a potential biomarker for acute myocardial infarction. Ann Transl Med. 2016;4(7):135.

21. Li R, Yan G, Li Q, et al. MicroRNA-145 protects cardiomyocytes against hydrogen peroxide $\left(\mathrm{H}_{2} \mathrm{O}_{2}\right)$-induced apoptosis through targeting the mitochondria apoptotic pathway. PLoS One. 2012;7(9):e44907.

22. Wang K, Liu F, Zhou LY, et al. The long noncoding RNA CHRF regulates cardiac hypertrophy by targeting miR-489. Circ Res. 2014;114(9): 1377-1388.

23. Wang K, Long B, Zhou LY, et al. CARL IncRNA inhibits anoxia-induced mitochondrial fission and apoptosis in cardiomyocytes by impairing miR-539-dependent PHB2 downregulation. Nat Commun. 2014;5:3596.

24. Yang J, Chen L, Ding J, et al. Cardioprotective effect of miRNA-22 on hypoxia/reoxygenation induced cardiomyocyte injury in neonatal rats. Gene. 2015;579(1):17-22.

25. Liu Y, Li G, Lu H, et al. Expression profiling and ontology analysis of long noncoding RNAs in post-ischemic heart and their implied roles in ischemia/reperfusion injury. Gene. 2014;543(1):15-21.

26. Liu Y, Zhou D, Li G, et al. Long non coding RNA-UCA1 contributes to cardiomyocyte apoptosis by suppression of p27 expression. Cell Physiol Biochem. 2015;35(5):1986-1998.

27. Michalik KM, You X, Manavski Y, et al. Long noncoding RNA MALAT1 regulates endothelial cell function and vessel growth. Circ Res. 2014; 114(9):1389-1397.

28. Doulamis IP, Tzani Al, Konstantopoulos PS, et al. A sirtuin 1/MMP2 prognostic index for myocardial infarction in patients with advanced coronary artery disease. Int J Cardiol. 2017;230:447-453.

29. Rayner BS, Duong TTH, Myers SJ, Witting PK. Protective effect of a synthetic anti-oxidant on neuronal cell apoptosis resulting from experimental hypoxia re-oxygenation injury. J Neurochem. 2006; 97(1):211-221.

30. Hartel FV, Holl MM, Aslam M, et al. Transient hypoxia induces ERKdependent anti-apoptotic cell survival in endothelial cells. Am JPhysiol Cell Physiol. 2010;298(6):1501-1509.
31. Bhogal RH, Weston CJ, Curbishley SM, Bhatt AN, Adams DH, Afford SC. Variable responses of small and large human hepatocytes to hypoxia and hypoxia/reoxygenation (H-R). FEBS Letters. 2011;585(6):935-941.

32. Hess ML, Barnhart GR, Crute S, Komwatana P, Krause S, Greenfield LJ. Mechanical and biochemical effects of transient myocardial ischemia. J Surg Res. 1979;26(2):175-184.

33. Bolli R, Marbán E. Molecular and cellular mechanisms of myocardial stunning. Physiol Rev. 1999;79(2):609-634.

34. Qiu MT, Hu JW, Yin R, Xu L. Long noncoding RNA: An emerging paradigm of cancer research. Tumour Biol. 2013;34(2):613-620.

35. Lorenzen JM, Thum T. Long noncoding RNAs in kidney and cardiovascular diseases. Nat Rev Nephrol. 2016;12(6):360-373.

36. Zhang M, Gu H, Xu W, Zhou X. Down-regulation of IncRNA MALAT1 reduces cardiomyocyte apoptosis and improves left ventricular function in diabetic rats. Int J Cardiol. 2016;203:214-216.

37. Wang X, Li M, Wang Z, et al. Silencing of long noncoding RNA MALAT1 by miR-101 and miR-217 inhibits proliferation, migration, and invasion of esophageal squamous cell carcinoma cells. J Biol Chem. 2015; 290(7):3925

38. Menghini R, Casagrande V, Cardellini M, et al. MicroRNA 217 modulates endothelial cell senescence via silent information regulator 1. Circulation. 2009;120(15):1524-1532.

39. Guarani V, Deflorian G, Franco CA, et al. Acetylation-dependent regulation of endothelial Notch signalling by the SIRT1 deacetylase. Nature. 2011;473(7346):234-238.

40. Nakahata Y, Sahar S, Astarita G, Kaluzova M, Sassone-Corsi P. Circadian control of the NAD+ salvage pathway by CLOCK-SIRT1. Science. 2009;324(5927):654-657.

41. Picard F, Kurtev M, Chung N, et al. Sirt1 promotes fat mobilization in white adipocytes by repressing PPAR-gamma. Nature. 2004; 429(6993):771-776.

42. Cho SH, Chen JA. SIRT1 deficiency in microglia contributes to cognitive decline in aging and neurodegeneration via epigenetic regulation of IL-1beta. J Neurosci. 2015;35(2):807-818.

43. Nadtochiy SM, Yao H, McBurney MW, et al. SIRT1-mediated acute cardioprotection. Am J Physiol Heart Circ Physiol. 2011;301(4): H1506-1512.

44. Hsieh-Cheng Chen MS, Yung-Ming Jeng MD, PhD. SIRT1 promotes tumorigenesis and resistance to chemotherapy in hepatocellular carcinoma and its expression predicts poor prognosis. Ann Surg Oncol. 2012;19(6):2011-2019.

45. Yang J, Wang N, Zhu Y, Feng P. Roles of SIRT1 in high glucose-induced endothelial impairment: Association with diabetic atherosclerosis. Arch Med Res. 2011;42(5):354-360.

46. Li W, Du D, Wang H, et al. Silent information regulator 1 (SIRT1) promotes the migration and proliferation of endothelial progenitor cells through the PI3K/Akt/eNOS signaling pathway. Int J Clin Exp Pathol. 2015;8(3):2274-2287. 